\title{
Existentially Definable Factor Congruences
}

\author{
Pedro Sánchez Terraf*
}

\begin{abstract}
A variety $\mathcal{V}$ has definable factor congruences if and only if factor congruences can be defined by a first-order formula $\Phi$ having central elements as parameters. We prove that if $\Phi$ can be chosen to be existential, factor congruences in every algebra of $\mathcal{V}$ are compact.
\end{abstract}

We study factor congruences in order to understand direct product representations in varieties. It is known that in rings with identity and bounded lattices, factor congruences are characterized, respectively, by central idempotent elements and neutral complemented elements. D. Vaggione [4] generalized these concepts to a broader context. A variety with $\overrightarrow{0} \& \overrightarrow{1}$ is a variety $\mathcal{V}$ in which there exist unary terms $0_{1}(w), \ldots, 0_{l}(w), 1_{1}(w), \ldots, 1_{l}(w)$ such that

$$
\mathcal{V} \models \overrightarrow{0}(w)=\overrightarrow{1}(w) \rightarrow x=y,
$$

where $w, x$ and $y$ are distinct variables, $\overrightarrow{0}=\left(0_{1}, \ldots, 0_{l}\right)$ and $\overrightarrow{1}=\left(1_{1}, \ldots, 1_{l}\right)$. If $\lambda \in A \in \mathcal{V}$, we say that $\vec{e} \in A^{l}$ is a $\lambda$-central element of $A$ if there exists an isomorphism $A \rightarrow A_{1} \times A_{2}$ such that

$$
\begin{gathered}
\lambda \mapsto\left\langle\lambda_{1}, \lambda_{2}\right\rangle, \\
\vec{e} \mapsto\left[\overrightarrow{0}\left(\lambda_{1}\right), \overrightarrow{1}\left(\lambda_{2}\right)\right] .
\end{gathered}
$$

where we write $[\vec{a}, \vec{b}]$ in place of $\left(\left\langle a_{1}, b_{1}\right\rangle, \ldots,\left\langle a_{l}, b_{l}\right\rangle\right) \in(A \times B)^{l}$ for $\vec{a} \in A^{l}$ and $\vec{b} \in B^{l}$. It is clear from the above definitions that if the language of $\mathcal{V}$ has a constant symbol $c$, the terms $\overrightarrow{0}$ and $\overrightarrow{1}$ can be chosen closed, and we can define a central element of $A$ to be just a $c^{A}$-central element. We will work heretofore under this assumption.

In [3], Vaggione and the author introduced the following concept:

Definition 1. $\mathcal{V}$ has Definable Factor Congruences (DFC) iff there exists a first order formula $\Phi(x, y, \vec{z})$ in the language of $\mathcal{V}$ such that for all $A, B \in \mathcal{V}$, and $a, c \in A, b, d \in B$,

$$
A \times B \models \Phi(\langle a, b\rangle,\langle c, d\rangle,[\overrightarrow{0}, \overrightarrow{1}]) \quad \text { if and only if } \quad a=c .
$$

*Supported by CONICET

2000 Mathematics Subject Classification: Primary 08B05, Secondary 03C40. 
Varieties with $\overrightarrow{0} \& \overrightarrow{1}$ are obviously semidegenerate (no non-trivial algebra in the variety has a trivial subalgebra). In [3] it is proved that DFC is equivalent to Boolean factor congruences [6, 2, 1] in semidegenerate varieties.

It is then natural to ask if, for a variety $\mathcal{V}$ with DFC, the quantifier complexity of $\Phi$ is reflected in the structure of $\mathcal{V}$. One early work [5] showed that if factor congruences in $\mathcal{V}$ are compact, then $\mathcal{V}$ has DFC and $\Phi$ can be chosen to be a positive existential formula. A partial converse was proved in [3]: if a positive formula $\Phi$ witnesses DFC for $\mathcal{V}, \mathcal{V}$ has compact factor congruences. Also, counterexamples were constructed showing that for universal $\Phi$ this may not be the case.

In this note we prove:

Theorem 2. Let $\mathcal{V}$ be a variety with $\overrightarrow{0} \& \overrightarrow{1}$. Suppose there exists an existential formula $\Phi$ that satisfies (1). Then we may replace $\Phi$ by a positive formula.

By using the results cited from [3] and [5] we obtain

Corollary 3. Let $\mathcal{V}$ be any variety. The following are equivalent:

1. There exist unary terms $\overrightarrow{0}(w)=0_{1}(w), \ldots, 0_{l}(w), \overrightarrow{1}(w)=1_{1}(w), \ldots, 1_{l}(w)$ and an existential first-order formula $\Phi(x, y, \vec{z})$ in the language of $\mathcal{V}$ such that for all $A, B \in \mathcal{V}$, and $a, c, e \in A, b, d, f \in B$,

$$
A \times B \models \Phi(\langle a, b\rangle,\langle c, d\rangle,[\overrightarrow{0}(e), \overrightarrow{1}(f)]) \quad \text { if and only if } \quad a=c .
$$

2. $\mathcal{V}$ has compact factor congruences.

Proof of Theorem 2. We will only consider the case $l=1$, so we have two closed terms 0 and 1 that satisfy

$$
A \times B \models \Phi(\langle a, b\rangle,\langle c, d\rangle,\langle 0,1\rangle) \quad \text { if and only if } \quad a=c .
$$

The general case is straightforward.

We will write $F\left(x_{1}, \ldots, x_{n}\right)$ for the algebra freely generated by $\left\{x_{1}, \ldots, x_{n}\right\}$ in $\mathcal{V}$. Assume

$$
\Phi(x, y, z):=\exists \vec{w} \bigvee_{i} \bigwedge_{j} \varphi_{i j}(x, y, z, \vec{w})
$$

where $\varphi_{i j}(x, y, z, \vec{u})$ is atomic or negated atomic and let $\Lambda_{i}=\left\{j: \varphi_{i j}\right.$ is atomic $\}$. Taking $A:=F(x)$ and $B:=F(x, y)$ in (2) we obtain:

$$
F(x) \times F(x, y) \models \exists \vec{w} \Phi(\langle x, x\rangle,\langle x, y\rangle,\langle 0,1\rangle, \vec{w})
$$

Hence there exists $[\vec{u}(x), \vec{v}(x, y)]$ in $F(x) \times F(x, y)$ and $k$ such that

$$
F(x) \times F(x, y) \models \bigwedge_{j} \varphi_{k j}(\langle x, x\rangle,\langle x, y\rangle,\langle 0,1\rangle,[\vec{u}(x), \vec{v}(x, y)])
$$


Using preservation by homomorphic images, we obtain

$$
\begin{aligned}
& \mathcal{V} \models \bigwedge_{j \in \Lambda_{k}} \varphi_{k j}(x, x, 0, \vec{u}(x)) \\
& \mathcal{V} \models \bigwedge_{j \in \Lambda_{k}} \varphi_{k j}(x, y, 1, \vec{v}(x, y))
\end{aligned}
$$

Now we will prove that for this $k$, the positive formula

$$
\Phi^{\prime}(x, y, z):=\exists \vec{w} \bigwedge_{j \in \Lambda_{k}} \varphi_{k j}(x, y, z, \vec{w})
$$

satisfies (2).

$(\Leftarrow)$ Take $a \in A, b, c \in B, A, B \in \mathcal{V}$. Using (4) and preservation by direct products,

$$
A \times B \models \bigwedge_{j \in \Lambda_{k}} \varphi_{k j}(\langle a, b\rangle,\langle a, c\rangle,\langle 0,1\rangle,[\vec{u}(a), \vec{v}(b, c)]) .
$$

Hence

$$
A \times B \models \exists \vec{w} \bigwedge_{j \in \Lambda_{k}} \varphi_{k j}(\langle a, b\rangle,\langle a, c\rangle,\langle 0,1\rangle, \vec{w})
$$

and by definition,

$$
A \times B \models \Phi^{\prime}(\langle a, b\rangle,\langle a, c\rangle,\langle 0,1\rangle)
$$

$(\Rightarrow)$ Now suppose $A \times B \models \Phi^{\prime}(\langle a, b\rangle,\langle c, d\rangle,\langle 0,1\rangle)$. By preservation by homomorphic images, we have $A \models \Phi^{\prime}(a, c, 0)$; take $\vec{w}$ such that

$$
A \models \bigwedge_{j \in \Lambda_{k}} \varphi_{k j}(a, c, 0, \vec{w}) .
$$

Considering (recall (3) )

$$
F(x) \times F(x, y) \models \bigwedge_{j \notin \Lambda_{k}} \varphi_{k j}(\langle x, x\rangle,\langle x, y\rangle,\langle 0,1\rangle,[\vec{u}(x), \vec{v}(x, y)])
$$

we obtain

$$
A \times(F(x) \times F(x, y)) \models \bigwedge_{j \notin \Lambda_{k}} \varphi_{k j}(\langle a,\langle x, x\rangle\rangle,\langle c,\langle x, y\rangle\rangle,\langle 0,\langle 0,1\rangle\rangle,[\vec{w},[\vec{u}(x), \vec{v}(x, y)]]),
$$

and hence, using (3) and (5),

$$
A \times(F(x) \times F(x, y)) \models \bigwedge_{j} \varphi_{k j}(\langle a,\langle x, x\rangle\rangle,\langle c,\langle x, y\rangle\rangle,\langle 0,\langle 0,1\rangle\rangle,[\vec{w},[\vec{u}(x), \vec{v}(x, y)]]) .
$$


Equivalently, by the obvious isomorphism

$$
(A \times F(x)) \times F(x, y) \models \bigwedge_{j} \varphi_{k j}(\langle\langle a, x\rangle, x\rangle,\langle\langle c, x\rangle, y\rangle,\langle\langle 0,0\rangle, 1\rangle,[[\vec{w}, \vec{u}(x)], \vec{v}(x, y)]) .
$$

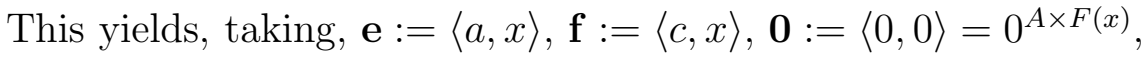

$$
(A \times F(x)) \times F(x, y) \models \Phi(\langle\mathbf{e}, x\rangle,\langle\mathbf{f}, x\rangle,\langle\mathbf{0}, 1\rangle)
$$

and by (2),

$$
A \times F(x) \models \mathbf{e}=\mathbf{f} .
$$

I.e., $\langle a, x\rangle=\langle c, x\rangle$. We may conclude $a=c$, as desired.

\section{References}

[1] C. C. Chang, B. Jónsson And A. TARski, Refinement properties for relational structures. Fund. Math. 54 (1964): 249-281.

[2] R. McKenzie, G. McNulty and W. Taylor, Algebras, Lattices, Varieties, Volume 1, The Wadsworth \& Brooks/Cole Math. Series, Monterey, California (1987).

[3] P. Sánchez Terraf and D. Vaggione, Varieties with Definable Factor Congruences, Trans. Amer. Math. Soc. 361 (2009): 5061-5088.

[4] D. VaGgione, $\mathcal{V}$ with factorable congruences and $\mathcal{V}=\mathbf{I} \Gamma^{a}\left(\mathcal{V}_{D I}\right)$ imply $\mathcal{V}$ is a discriminator variety. Acta Sci. Math. 62 (1996): 359-368.

[5] D. Vaggione and P. SÁnchez Terraf, Compact factor congruences imply Boolean factor congruences, Algebra univers. 51 (2004): 207-213.

[6] R. Willard, Varieties Having Boolean Factor Congruences. J. Algebra, 132 (1990): 130153.

CIEM - Facultad de Matemática, Astronomía y Física (Fa.M.A.F.)

Universidad Nacional de Córdoba - Ciudad Universitaria

Córdoba 5000. Argentina.

sterraf@famaf .unc.edu.ar 